\title{
Towards human central nervous system in vitro models for preclinical research: strategies for 3D neural cell culture
}

\author{
Daniel Simão $0^{1,2}$, Inês Costa ${ }^{1,2}$, Margarida Serra ${ }^{1,2}$, Johannes Schwarz ${ }^{3}$, Catarina Brito ${ }^{1,2^{*}}$, Paula M Alves ${ }^{1,2}$ \\ From 22nd European Society for Animal Cell Technology (ESACT) Meeting on Cell Based Technologies \\ Vienna, Austria. 15-18 May 2011
}

\section{Background}

The development of new drugs for human Central Nervous System (CNS) diseases has traditionally relied on 2D in vitro cell models and genetically engineered animal models. However, those models often diverge considerably from that of human phenotype (anatomical, developmental and biochemical differences) [1] contributing to a high attrition rate - only $8 \%$ of CNS drugs entering clinical trials end up being approved [2]. Human 3D in vitro models are useful complementary tools towards more accurate evaluation of drug candidates in pre-clinical stages, as they present an intermediate degree of complexity in terms of cell-cell and cellmatrix interactions, between the traditional 2D monolayer culture conditions and the complex brain and can be a better starting point for the analysis of the in vivo context. Aiming at developing novel $3 \mathrm{D}$ in vitro models of the CNS, this work focus on the implementation of long-term cultures of human midbrain-derived neural stem cells (hmNSC) for the scalable supply of neuralsubtype cells, with a focus on the dopaminergic lineage, following a systematic technological approach based on stirred culture systems.

\section{Materials and methods}

Cell culture: hmNSC were isolated as previously reported [3] and routinely propagated in static conditions, on poly-L-ornithine-fibronectin (PLOF) coated plates, in serum-free propagation medium, containing basic fibroblast growth factor and epidermal growth factor [3]. hmNSC were cultured in stirred systems in Cultispher $\mathrm{S}$ microcarriers (Percell Biolytica) without

'Instituto de Tecnologia Química e Biológica - Universidade Nova de Lisboa, 2780-157 Oeiras, Portugal

Full list of author information is available at the end of the article coating and coated with PLOF) or as neurospheres for 7 to 21 days, with media changes every 3-4 days. All experiments were performed in $125 \mathrm{~mL}$ shake flasks (20 $\mathrm{mL}$ working volume), with orbital shaking at $100 \mathrm{rpm}$. Cultures were maintained at $37^{\circ} \mathrm{C}$, in $3 \% \mathrm{O}_{2}$. Double stain viability test: aggregates were collected from stirred cultures, incubated with fluorescein diacetate $(10 \mu \mathrm{g} /$ $\mathrm{mL})$ and propidium iodide $(1 \mu \mathrm{g} / \mathrm{mL})$ and observed on a fluorescence microscope (Leica DMI6000). Aggregate size was measured in pictures taken from each culture sample using Image J software (NIH), as previously reported [4]. Dissociation: For microcarrier cultures, Cultispher S was allowed to settle, washed with PBS and digested with Trypsin 0.05\%-EDTA (Gibco). Cells were collected by centrifugation and counted by trypan blue exclusion dye. Free cells were counted using the same aliquot. Aggregates were dissociated with Accutase (Sigma).

\section{Results}

The feasibility of culturing hmNSC as 3D structures in stirred culture systems was assessed by testing two different approaches: microcarrier technology versus cell aggregated cultures (neurospheres). For the first strategy, Cultispher S, a collagen-based macroporous microcarrier was tested for its ability to support hmNSC attachment and growth. Microcarriers uncoated and coated with PLOF were tested. hmNSCs were labelled with PKH26 lipophilic dye (red) for detection purposes and inoculated at $1.5 \times 10^{5} \mathrm{cell} / \mathrm{mL}$, in a carrier concentration of $1 \mathrm{~g} / \mathrm{L}$, corresponding to approximately $125 \mathrm{cell} /$ microcarrier. Monitoring along 5 days of culture time revealed that the fraction of viable cells found on the microcarriers was less than $10 \%$ of the inoculum, for both uncoated and PLOF-coated microcarriers, indicating 


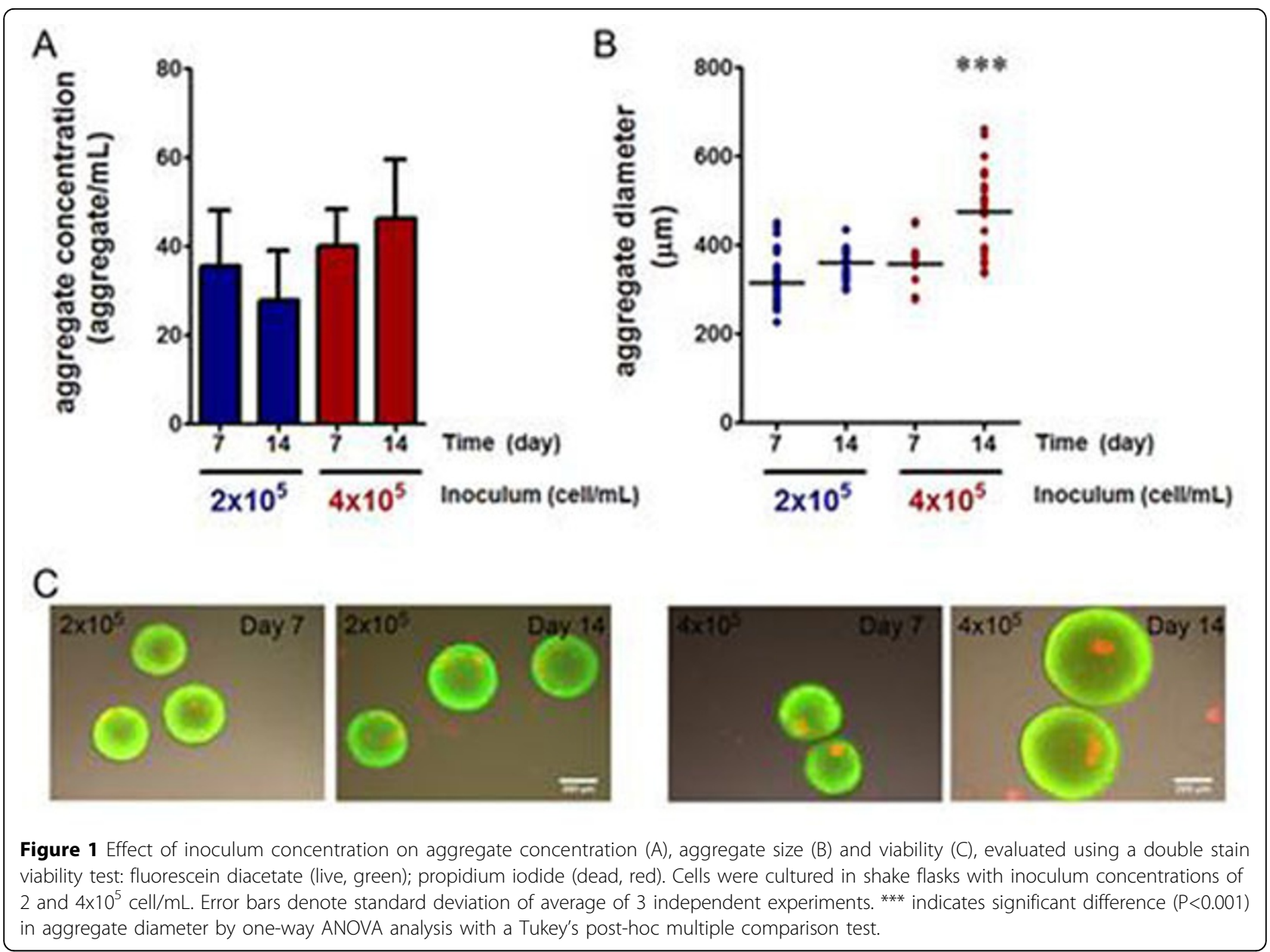

poor microcarrier colonization. Fluorescence microscopy analysis revealed that hmNSC aggregated in suspension rather than colonizing Cultispher S microcarriers (not shown). For the cell aggregate strategy, two inoculum concentrations were tested -2 and $4 \times 10^{5}$ cell $/ \mathrm{mL}$ and aggregate size and number evaluated along culture time (Figure 1).

The inoculum concentration of $2 \times 10^{5} \mathrm{cell} / \mathrm{mL}$ was as efficient as $4 \times 10^{5}$ cell $/ \mathrm{mL}$ in promoting cell aggregation (Figure 1A) whereas it allowed for lower mean diameters along culture time $(362 \pm 32 \mu \mathrm{m}$ at day 14$)$ as compared to the higher inoculum concentration for which significantly higher mean diameter and also a wider range of aggregate sizes were observed (475 \pm 103 $\mu \mathrm{m}$ at day 14) (Figure 1B). Moreover, the lower inoculum concentration avoided the formation of necrotic centres, which were detected in cultures with an inoculum concentration of $4 \times 10^{5}$ cell $/ \mathrm{mL}$ (Figure 1C).Taken together the data presented indicates that $2 \times 10^{5}$ cell $/ \mathrm{mL}$ is the most favourable inoculum concentration for culture of hmNSC as aggregates in stirred culture systems.

\section{Conclusions}

In this study the feasibility of culturing hmNSC as 3D structures in stirred culture systems was evaluated. Cell aggregates (neurosphere) culture, using an inoculum concentration of $2 \times 10^{5} \mathrm{cell} / \mathrm{mL}$ was selected as the best strategy, due to the higher cell viabilities and tightly control of aggregate diameter attained. The implemented 3D culture system will be applied in the optimization of differentiation of hmNSC into dopaminergic neurons, astrocytes and oligodendrocytes.

\section{Acknowledgments}

The authors acknowledge the FP7 EU project BrainCAV (HEALTHHS_2008_222992) and the FCT project PTDC/EBB-BIO/112786/2009 for financial support.

\section{Author details}

${ }^{1}$ Instituto de Tecnologia Química e Biológica - Universidade Nova de Lisboa, 2780-157 Oeiras, Portugal. ${ }^{2}$ Instituto de Biologia Experimental e Tecnológica, 2780-901 Oeiras, Portugal. ${ }^{3}$ Department of Neurology, University of Leipzig, 04103 Leipzig, Germany.

Published: 22 November 2011 


\section{References}

1. Gagliardi C, Bunnell BA: Large animal models of neurological disorders for gene therapy. ILAR J 2009, 50:128-143.

2. Miller G: Is pharma running out of brainy ideas? Science 2010, 329:502-504.

3. Milosevic J, Schwarz SC, Ogunlade V, Meyer AK, Storch A, Schwarz J: Emerging role of LRRK2 in human neural progenitor cell cycle progression, survival and differentiation. Mol Neurodegener 2009, 4:25.

4. Serra M, Brito C, Costa E, Sousa MFQ, Alves PM: Integrating human stem cell expansion and neuronal differentiation in bioreactors. $B M C$ Biotechnology 2009, 8:92.

doi:10.1186/1753-6561-5-S8-P53

Cite this article as: Simão et al:: Towards human central nervous system in vitro models for preclinical research: strategies for $3 \mathrm{D}$ neural cell culture. BMC Proceedings 2011 5(Suppl 8):P53.

\section{Submit your next manuscript to BioMed Central} and take full advantage of:

- Convenient online submission

- Thorough peer review

- No space constraints or color figure charges

- Immediate publication on acceptance

- Inclusion in PubMed, CAS, Scopus and Google Scholar

- Research which is freely available for redistribution

Submit your manuscript at www.biomedcentral.com/submit 